\title{
Spaceflight and Biohistochemical Alterations of Antigravity Soleus Muscle in Spontaneously Hypertensive Rats (SHR) to Dietary Low- and High-
} Calcium Intake

\section{Sadayoshi Taguchi ${ }^{*}$, Shigehiko Ogoh ${ }^{2}$, Takeshi Hashimoto ${ }^{1}$, Sakiya Yamasaki ${ }^{3}$, Hiroshi Okamoto ${ }^{4}$, Yukio Yamori ${ }^{5}$, Daniel C Hatton $^{6,7}$ and David A McCarron ${ }^{8}$}

${ }^{1}$ Graduate School of Sport and Health Sciencelth Science, Ritsumeikan University, Kusatsu, Japan

${ }^{2}$ Department of Biomedical Engineering, Toyo University, Saitama, Japan

${ }^{3}$ Graduate School of Medicine and Pharmaceutical Sciences for Research, University of Toyama, Toyama, Japan

${ }^{4}$ Department of Liberal Arts and Sciences, Toyama Prefectural University, Toyama, Japan

5Institute for World Health Development, Mukogawa Women's University, Nishinomiya, Japan

${ }^{6}$ Department of Behavioral Neuroscience, Oregon Health Sciences University, Portland, USA

${ }^{7}$ Divisions of Nephrology, Hypertension, and Clinical Pharmacology, Oregon Health Sciences University, Portland, USA

${ }^{8}$ Department of Nutrition, University of California at Davis, Davis, CA, USA

\begin{abstract}
Dietary calcium supplementation has been used to alter calcium metabolism and prevent hypertension in spontaneously hypertensive rats (SHR). Likewise, microgravity alters calcium metabolism. It is possible that the alteration of calcium metabolism caused by different levels of dietary calcium or microgravity change skeletal muscle properties. The purpose of this investigation was to determine whether spaceflight and/or dietary calcium causes alteration of properties in skeletal muscle from SHR.

Nine-week-old male SHR, fed either high- $(2.0 \%, \mathrm{HCa})$ or low-calcium $(0.2 \%$, LCa) diets, were flown on an 18-day shuttle flight. They were assigned to four groups ( $n=7 /$ group): control (ground-based) with LCa or $\mathrm{HCa}$, spaceflight with $\mathrm{LCa}$, or $\mathrm{HCa}$. After the spaceflight, the soleus muscle was dissected and analyzed histochemically and biochemically.

Spaceflight caused a slow-to-fast fiber transition that did not differ between diet groups. Cross-sectional areas of a single muscle fiber were not modified by dietary calcium in either control or spaceflight groups. The changes of succinate dehydrogenase activity in Type I fibers in response to spaceflight was higher in the LCa diet (+23.7\%) than the HCa diet group $(+12.8 \%)$. Conversely, in Type lla fibers the response to spaceflight was lower in the LCa $(-9.6 \%)$ than the $\mathrm{HCa}(-19.2 \%)$ group. These findings suggest that the effect of dietary calcium on fiber type composition and fiber size may be smaller than that of spaceflight, while dietary calcium may inhibit the alteration of oxidative metabolism of muscle fibers by spaceflight in SHR.
\end{abstract}

Keywords: SHR; Calcium diet level; Skeletal muscle; Spaceflight

\section{Introduction}

Previous studies have reported that skeletal muscle characteristics are altered with cardiovascular risk factors and suggest that these characteristics may have important health-related implications [1]. Benbachir-Lamrini et al., [2,3] and Lewis et al., [4] reported that soleus muscles from spontaneously hypertensive rats (SHR) contain fewer type I fibers and more type II fibers than soleus of age-matched normotensive Wistar Kyoto (WKY) rats. In addition, muscle biopsy findings in patients with chronic heart failure showed a reduced proportion of slow-twitch oxidative fibers and decreased oxidative enzyme activity [5].

On the other hand, the alterations of skeletal muscle in patients with cardiovascular disease also resemble those that occur with disuse. Many previous investigations of bed-rest or spaceflight have demonstrated weightlessness to result in alterations of structural and metabolic profiles of skeletal muscle. However, whether these changes in skeletal muscle properties in patients result from disuse or occur as a direct consequence of factors related to cardiovascular disease remains unclear.

Bone-free lean mass is correlated with bone mineral variables and important predictor of bone size in various populations [6-8]. In our animal study, Ogoh et al., [9] showed that atrophy and slowto-fast fiber type transition in SHR were caused by body suspension, and there was a strong correlation between muscle weight and bone calcium content $(y=-360.54+23.781 x, r=0.818)$. In this regard, Ingalls et al., showed that the resting $\mathrm{Ca}^{2+}$ levels increase in soleus fibers of hindlimb unloaded mice [10]. Pardo et al., found that mitochondrial $\mathrm{Ca}^{2+}$ uptake and accumulation capacity of SHR are lower compared to normotensive rats [11]. However, Shenkman and Nemirovskaya reported that nifedipine (L-type $\mathrm{Ca}^{2+}$ channels blocker) administration was shown to considerably restrict the slowto-fast transition of myosin heavy chains (MHC) under unloading (at the RNA level and the protein level as well) based on their study $[12,13]$. On the other hand, $\mathrm{Ca}^{2+}$ ions also activate four mitochondrial enzymes that are involved in substrate dehydrogenation and production of NADH [14]. These results suggested the possibility that alterations of $\mathrm{Ca}^{2+}$ metabolism after spaceflight influence skeletal muscle properties.

*Corresponding authors: Sadayoshi Taguchi, Graduate School of Sport and Health Science, Ritsumeikan University, 1-1-1 Nojihigashi, Kusatsu, 525-8577, Japan, Tel: +81-90-3991-9614; E-mail: staguchi290@gmail.com

Received May 20, 2016; Accepted May 31, 2016; Published June 07, 2016

Citation: Taguchi S, Ogoh S, Hashimoto T, Yamasaki S, Okamoto H, et al. (2016) Spaceflight and Biohistochemical Alterations of Antigravity Soleus Muscle in Spontaneously Hypertensive Rats (SHR) to Dietary Low and High-Calcium Intake. J Bioanal Biomed 8: 041-047. doi:10.4172/1948-593X.1000151

Copyright: ( 2016 Taguchi S, et al. This is an open-access article distributed under the terms of the Creative Commons Attribution License, which permits unrestricted use, distribution, and reproduction in any medium, provided the original author and source are credited. 
Citation: Taguchi S, Ogoh S, Hashimoto T, Yamasaki S, Okamoto H, et al. (2016) Spaceflight and Biohistochemical Alterations of Antigravity Soleus Muscle in Spontaneously Hypertensive Rats (SHR) to Dietary Low and High-Calcium Intake. J Bioanal Biomed 8: 041-047. doi:10.4172/1948593X.1000151

In hypertensive studies, an abundance of evidence has shown that variations in calcium intake and metabolism are associated with significant differences in blood pressure $[15,16]$. Hatton et al., demonstrated that low-calcium diets reduce ionized calcium levels in rats exposed to spaceflight, causing an elevation of parathyroid hormone $(\mathrm{PTH})$ and $1.25(\mathrm{OH})_{2} \mathrm{D}_{3}$ to correct the deficit [17]. McCarron et al., showed that lower calcium intake was the most consistent factor in hypertensive individuals [18]. Ono et al., also found that dietary calcium supplementation prevented accelerated hypertension with sympathetic over activity as well as impairment of the arterial baroreceptor reflex [19].

Taken together, these reports suggested the possibility that the alteration of calcium metabolism caused by different level of dietary calcium or microgravity change the mitochondrial function and oxidative capacity in skeletal muscle. The purpose of this investigation was to determine 1) whether disuse (spaceflight) causes alteration of properties in skeletal muscle from SHR, 2) whether the properties in skeletal muscle from SHR are altered by different level of dietary calcium, and 3) whether the alteration of skeletal muscle from SHR after exposure to microgravity is impacted by different levels of dietary calcium.

\section{Research Design and Methods}

The data of skeletal muscle analysis presented in this report were obtained from NIH.R4, a life sciences mission flown on a NASA space shuttle (STS-80). That mission was designed to examine the influence of dietary calcium on calcium metabolism and cardiovascular function in microgravity [17]. The data presented here describe the effects of spaceflight on skeletal muscle characteristics in hypertension.

\section{Experiment animals}

An animal care protocol was approved by the institutional Animal Care and Use Committees at Oregon Health Sciences University, Ames Research Center, Johnson Space Center, and Kennedy Space Center. Twenty eight male spontaneously hypertensive rats (SHR), the most commonly used genetically hypertensive rat, were obtained from Taconic Farms (Germantown, NY). Nine-weeks (wks)-old SHR, fed either high or low-calcium diets (HCa or LCa, respectively), were flown on an 18-day shuttle flight. At 21 days of age, male SHRs were assigned to four groups ( $\mathrm{n}=7 /$ group): 1) control SHR with $\mathrm{LCa}, 2)$ control SHR with HCa, 3) spaceflight SHR with LCa, and 4) spaceflight SHR with $\mathrm{HCa}$. The animals were placed on either an $\mathrm{HCa}(2.0 \%)$ or $\mathrm{LCa}$ $(0.2 \%)$ diet (Teklad, Madison, WI) for the duration of the experiment. Calcium content was the only difference between the present diet and the typical space bar diet consumed by rodents on shuttle flights. The animals were assigned to flight and control groups based on receipt date from the vendor. Shipments were offset by 3 days to allow for testing of all animals at the same age. When the animals were 7-wks of age, two animal enclosure modules (AEM) containing seven animals each were flown on the space shuttle Columbia (STS-80), housed in controlled environment $\left(21 \pm 1^{\circ} \mathrm{C}\right)$. One AEM contained high-calcium diet and the other, low-calcium diet animals. The spaceflight rats were killed 7-14 h after return to $1 \mathrm{G}$. The control rats were killed on exactly the same schedule as that for the flight animals, but 3 days later. The soleus was excised from each rat, frozen in melting isopentane, and stored at $-80^{\circ} \mathrm{C}$ until analyzed.

\section{Histochemical analysis}

The muscles to be analyzed for the histochemical properties were sliced transversally in the mid-belly region into $10 \mu \mathrm{m}$ thick sections on a cryostat at $-20^{\circ} \mathrm{C}$. To determine muscle fiber type and calculate proportions for each fiber type as well as the cross-sectional areas (CSA) in individual muscle fibers, myofibrillar adenosine triphosphatase (ATPase) histochemical staining procedures were performed according to an established technique [20]. Muscle fibers were characterized as slow-twitch oxidative (Type I), fast-twitch oxidative glycolytic (Type IIa), and intermediate (Type IIx), which shares the histochemical profiles of Type I and Type IIa fibers [21]. Fiber type distribution was expressed as the number of fibers of each type relative to the total number of fibers (>200 fibers/section). The fiber cross-sectional areas (CSA) were calculated by use of an image processing system (LA-535FB, Pias, Osaka) coupled to a digitizer on ATP-stained sections. The adjacent sections were incubated in different media for succinate dehydrogenase $(\mathrm{SDH})[22]$ and alpha-glycerol phosphate dehydrogenase ( $\alpha-\mathrm{GPD})$.

\section{Image analysis for histochemical enzyme activity}

Images were captured by an optical microscope (Y2F-21, Nikon, Tokyo) with a connected CCD video camera (SSC-370, Sony, Tokyo) under the same microscope objective. An image processing system (LA-535FB, Pias, Osaka) was used to perform image analysis. To determine enzyme activity in individual muscle fibers of soleus muscle, the quantitative histochemical microphotometric procedure was used. First, muscle fibers were classified as fiber types based on the ATPase staining. Second, individual fibers identified on the slides prepared for fiber type determination were then reidentified on the sections stained for SDH and a-GPD to assess for oxidative and glycolytic enzyme activities. Each fiber was digitized with an individual picture element (pixel) equivalent to $0.4 \mu \mathrm{m}^{2}$ in area. These pixels were quantified to 255 gray levels in terms of brightness. The SDH and $\alpha$-GPD activities of a single muscle fiber were the values that were the mean gray level of all pixels included in a single muscle fiber. The mean value for each of these parameters in relation to fiber type was then calculated for each individual. At least four fields from each section were analyzed in collecting data for these determinations.

\section{Biochemical enzyme activity}

The enzyme activities were determined from 5-10\% homogenates of tissue in the medium ( $175 \mathrm{mM} \mathrm{KCl}, 10 \mathrm{mM} \mathrm{GSH}$, and $2 \mathrm{mM}$ EDTA $\mathrm{pH}$ 7.4). The homogenates were frozen and thawed several times to disrupt the mitochondrial membrane. Lactic dehydrogenase (LDH), citrate synthase (CS), and 3-hydroxyacyl-CoA dehydrogenase (HAD) activities were measured as previously described [23]. The enzyme activities are expressed as micromoles of substrate per minute per gram wet weight.

\section{Gel electrophoresis}

MHC (myosin heavy chain) isoforms were separated by sodiumdodecyl surfate polyacrylamide gel electrophoresis (SDS-PAGE) according to Talmadge and Roy [24]. The muscles were solubilized in $10 \mu \mathrm{l}$ of $2 \%$ SDS sample buffer (containing $62.5 \mathrm{mM}$ Tris-HCl, pH $6.8,25 \%$ glycerol, $2 \%$ SDS, $0.01 \%$ bromophenol blue, and $5 \%$ 2 -mercaptoethanol), and stored at $-80^{\circ} \mathrm{C}$. The compositions of the stacking and separating gels were 4 and $8 \%$ acrylamide, respectively. The gels were placed into an electrophoretic chamber (Bio-Rad Protean Iix). Electrophoresis was performed by using a constant voltage of $275 \mathrm{~V}$ for $24 \mathrm{~h}$. The SDS-PAGE gels were stained according to the procedures described by our previous study [25]. A computer-based image analysis system and software (Multi-Analyst, Bio-Rad) were used to quantify the relative density of each MHC isoform. 
Citation: Taguchi S, Ogoh S, Hashimoto T, Yamasaki S, Okamoto H, et al. (2016) Spaceflight and Biohistochemical Alterations of Antigravity Soleus Muscle in Spontaneously Hypertensive Rats (SHR) to Dietary Low and High-Calcium Intake. J Bioanal Biomed 8: 041-047. doi:10.4172/1948593X.1000151

\section{Statistical analysis}

The values were expressed as mean \pm the standard error of the mean (SE). The data were analyzed using a two-way analysis variance (ANOVA). If a significant F statistic was obtained, a student NewmanKeuls post hoc test was used to establish significant group mean differences. Analyses were conducted using SigmaStat (Jandel Scientific Software, SPSS Inc., Chicago, IL, USA). The significance level selected was $\mathrm{p}<0.05$

\section{Results}

\section{Fiber type distribution and MHC isoform composition}

Histochemical analysis of fiber type distributions for soleus muscle is presented in Figure 1. There was no significant difference in histochemical fiber type distribution of soleus muscle between $\mathrm{HCa}$ and LCa diet in either control or spaceflight SHR rats. However, the proportion of type I MHC isoform was significantly $(\mathrm{p}<0.005)$ higher in HCa diet group (79.2\%) than LCa diet group (74.0\%) before spaceflight (Figures 2 and 3) $(\mathrm{F}=5.46, \mathrm{p}<0.05)$. Conversely, the percentage of type IIa MHC isoform was significantly $(\mathrm{p}<0.05)$ lower in control $\mathrm{HCa}$ diet group (19.7\%) as compared with their LCa counterpart $(23.0 \%)(\mathrm{F}=7.72$, $\mathrm{p}<0.005)$. After spaceflight, the slow-to-fast fiber type transition was observed from both histochemical (Type I: $\mathrm{F}=20.92, \mathrm{p}<0.001$; Type IIa: $\mathrm{F}=11.02, \mathrm{p}<0.005$; Type IIx: $\mathrm{F}=5.88, \mathrm{p}<0.05$ ) and $\mathrm{MHC}$ isoform (type I MHC: $\mathrm{F}=480.35, \mathrm{p}<0.001$; type IIa MHC: $\mathrm{F}=20.02$, $\mathrm{p}<0.001$; type IIx MHC: $F=820.50, p<0.001$; type IIb MHC: $F=145.60, p<0.001$ ) analysis in both diet groups. There was a significant increase of Type IIa fibers, Type IIx and Type IIb MHC isoforms in both diet groups $(\mathrm{p}<0.05)$. In addition, changes in MHC isoform composition caused by spaceflight in the LCa diet group were not different from the $\mathrm{HCa}$ diet group (Figure 4A). Though we observed the difference in MHC composition between $\mathrm{HCa}$ and $\mathrm{LCa}$ in control SHR rats, there was no difference in $\mathrm{MHC}$ isoform composition between the HCa and LCa diet groups after spaceflight.

\section{The cross-sectional areas (CSA) in a single muscle fiber}

The CSA in a single muscle fiber of each group is presented in Table 1 . In these results, it is clear that muscle atrophy was caused by spaceflight in Type I fiber. After spaceflight, there was a significant reduction of CSA in both diet groups in Type I fiber $(F=35.79, p<0.001)$. In addition, reductions of CSA in the Type I fiber were greater than in the Type IIa fiber (Figure 4B). However, these atrophy levels of a single muscle fiber were not influenced by different dietary calcium in both the Type I ( -44.3 vs. $-41.3 \%$ for HCa vs. LCa) and the Type IIa (-4.6 vs. $-10.7 \%$ for HCa vs. LCa) fibers.

\section{Histochemical enzyme activity of a single muscle fiber}

Table 2 shows the histochemical SDH and alpha-GDP activities of a single muscle fiber. In both control and spaceflight SHR rats, the

\begin{tabular}{|c|c|c|c|}
\hline \multicolumn{2}{|c|}{} & \multicolumn{2}{|c|}{ CSA } \\
\cline { 3 - 4 } \multicolumn{2}{|c|}{} & Type I $\left(\boldsymbol{\mu m}^{2}\right)$ & Type Ila $\left(\boldsymbol{\mu m}^{2}\right)$ \\
\hline \multirow{2}{*}{ LCa } & CON & $2150 \pm 182$ & $1735 \pm 144$ \\
\cline { 2 - 4 } & SF & $1263 \pm 132^{\star}$ & $1549 \pm 136$ \\
\hline \multirow{2}{*}{ HCa } & CON & $1875 \pm 146$ & $1532 \pm 111$ \\
\cline { 2 - 4 } & SF & $1045 \pm 66^{*}$ & $1462 \pm 167$ \\
\hline
\end{tabular}

Values are means \pm SE. Significant difference between flight and control groups: 'P<0.05. Type I; slow-twitch oxidative fiber, Type Ila; fast-twitch oxidative glycolytic fiber.

Table 1: Cross-sectional area (CSA) of a single muscle fiber of soleus muscle for flight (SF) and control (CON) animals on high-(HCa) and low-calcium (LCa) diets.
SDH activities of both Type I and Type IIa fibers were lower in the HCa than the LCa diet groups (Type I: $F=19.01, \mathrm{p}<0.001$; Type IIa: $\mathrm{F}=36.38$, $\mathrm{p}<0.001)$. The SDH activity of Type I fiber was higher in the spaceflight than the control group in both the $\mathrm{HCa}$ and the $\mathrm{LCa}$ diet groups $(\mathrm{F}=13.04, \mathrm{p}<0.005)$. In addition, there were differences in these changes that were caused by spaceflight between $\mathrm{HCa}$ and LCa diet groups. The difference in SDH activity between the control and the spaceflight SHR rats was higher in the LCa diet (+23.7\%) than the HCa diet group $(+12.8 \%)$ (Figure $4 \mathrm{C})$. Conversely, the SDH activity of Type IIa fiber of soleus muscle was lower in the spaceflight rats than the control group in both the $\mathrm{HCa}(-19.2 \%)$ and the $\mathrm{LCa}(-9.6 \%)$ diet groups. The difference of SDH activity of Type IIa fiber between the control and the spaceflight SHR rats was lower in the LCa than the HCa diet group.

The $a$-GPD activities of both Type I and Type IIa fibers were increased after spaceflight in both the $\mathrm{HCa}$ and the LCa diet groups (Type I: $\mathrm{F}=8.23, \mathrm{p}<0.05$; Type IIa: $\mathrm{F}=12.82, \mathrm{p}<0.005$ ), but the difference was not significant.

\section{Discussion}

The accomplishment of the present investigation was the assessment of the skeletal muscle responses to spaceflight (disuse) or dietary calcium level in spontaneously hypertensive rats. In previous studies, it has been made clear that microgravity causes atrophy, reduction of mitochondrial enzyme activities and a shift of contractile properties from slow- to fast-twitch muscle fiber type in skeletal muscle [26-29]. It was also reported that hypertension alters muscle characteristics [1$4,30]$. Moreover, cardiovascular diseases decrease activity of patients in daily life. However, only a few studies [31] investigated the influence of the interaction between spaceflight, or disuse, and cardiovascular disease on skeletal muscle characteristics, although in the future, it is expected that cardiovascular patients will also go to space.

The major findings of the present investigation were that, 1) a significant effect of spaceflight on the elevation of fast twitch fibers in the number and also in MHC expression of type IIa and type IIx, and the amount of slow-twitch fiber atrophy was much more prominent than that of fast-twitch fibers, 2) there was no significant difference in the amount of atrophy of a single muscle fiber between $\mathrm{HCa}$ and LCa in both ground-based and spaceflight groups, 3) the oxidative enzyme activity was influenced by both spaceflight and the dietary calcium level. These changes in slow-twitch fiber were also different from fasttwitch fiber, and 4) the glycolytic enzyme activity was not influenced by either spaceflight or dietary calcium level.

\section{Fiber type distribution}

In the MHC isoform analysis, the proportion of type I MHC isoform was significantly higher in the HCa diet group when compared to the LCa diet group before spaceflight $(\mathrm{P}<0.005)$. The calcium diet

\begin{tabular}{|c|c|c|c|c|c|}
\hline \multicolumn{2}{|c|}{} & \multicolumn{2}{c|}{ SDH } & \multicolumn{2}{c|}{ a-GPD } \\
\cline { 2 - 6 } \multicolumn{2}{|c|}{} & Type I (O.D.) & Type Ila (O.D.) & Type I (O.D.) & Type Ila (O.D.) \\
\hline \multirow{2}{*}{ LCa } & CON & $80.5 \pm 3.1$ & $116.5 \pm 3.4$ & $42.9 \pm 6.0$ & $103.3 \pm 4.4$ \\
\cline { 2 - 6 } & SF & $99.5 \pm 4.1^{\star}$ & $105.3 \pm 5.7$ & $62.8 \pm 5.5^{\star}$ & $117.5 \pm 3.1$ \\
\hline \multirow{2}{*}{ HCa } & CON & $68.3 \pm 6.1$ & $90.2 \pm 5.1^{\dagger}$ & $49.1 \pm 7.5$ & $88.3 \pm 5.0$ \\
\cline { 2 - 6 } & SF & $77.0 \pm 2.8^{\dagger}$ & $72.9 \pm 3.8^{\star}$ & $61.3 \pm 4.0$ & $108.1 \pm 6.2$ \\
\hline
\end{tabular}

Values are means \pm SE. Significant difference between flight and control groups: ${ }^{*} \mathrm{P}<0.05$. Significant difference between diet groups: ${ }^{\dagger} \mathrm{P}<0.05$. Type I; slow-twitch oxidative fiber, Type lla; fast-twitch oxidative glycolytic fiber.

Table 2: Succinate dehydrogenase (SDH) and alpha-glycerol phosphate dehydrogenase ( $\alpha-G P D$ ) of a single muscle fiber of soleus muscle for flight (SF) and control (CON) animals on high-( $\mathrm{HCa})$ and low-calcium ( $\mathrm{LCa})$ diets. 


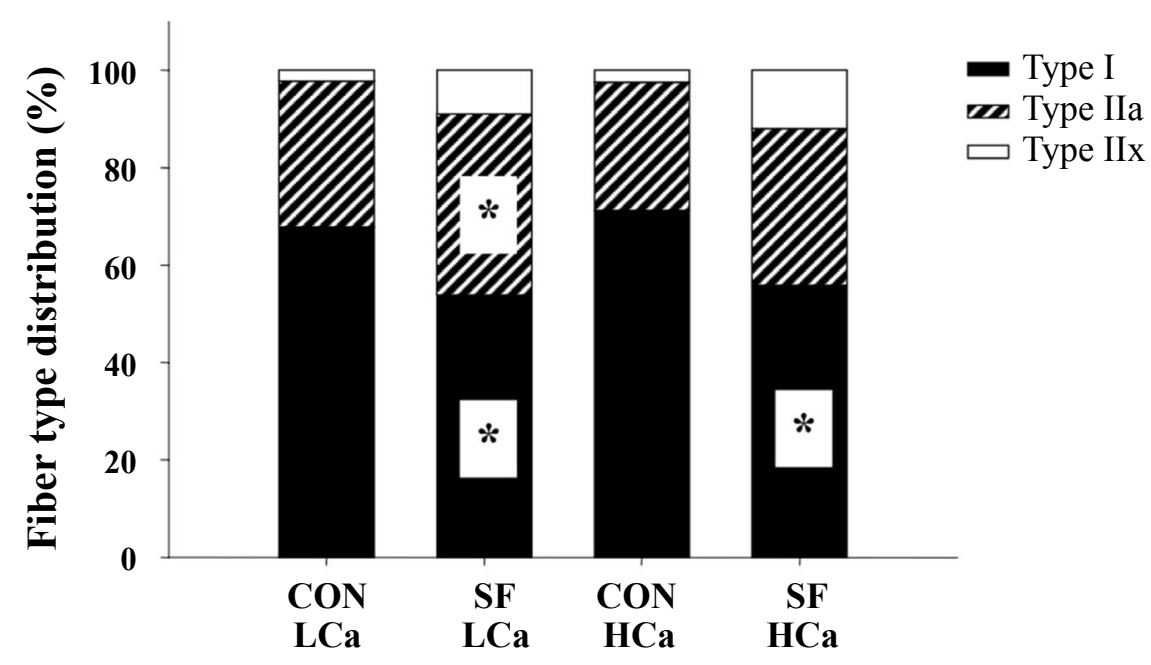

Figure 1: Histochemical fiber type distribution in soleus muscle for flight (SF) and control (CON) animals on high-(HCa) and low-calcium (LCa) diets. Significant difference between flight and control groups: " $\mathrm{P}<0.05$. Type I; slow-twitch oxidative fiber, Type Ila; fast-twitch oxidative glycolytic fiber, Type Ilx; intermediate fiber.

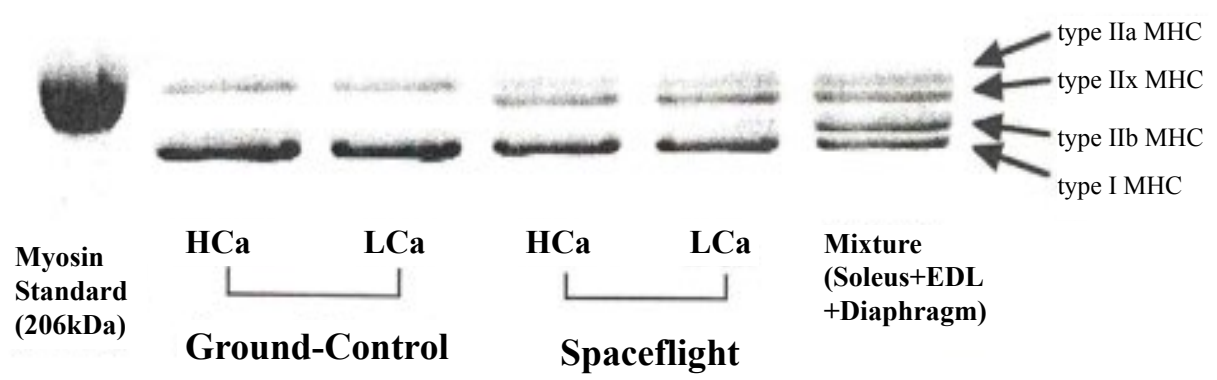

Figure 2: Electrophoretic separation of myosin heavy chain (MHC) isoforms in soleus muscle for flight and control animals on high-(HCa) and low-calcium (LCa) diets.

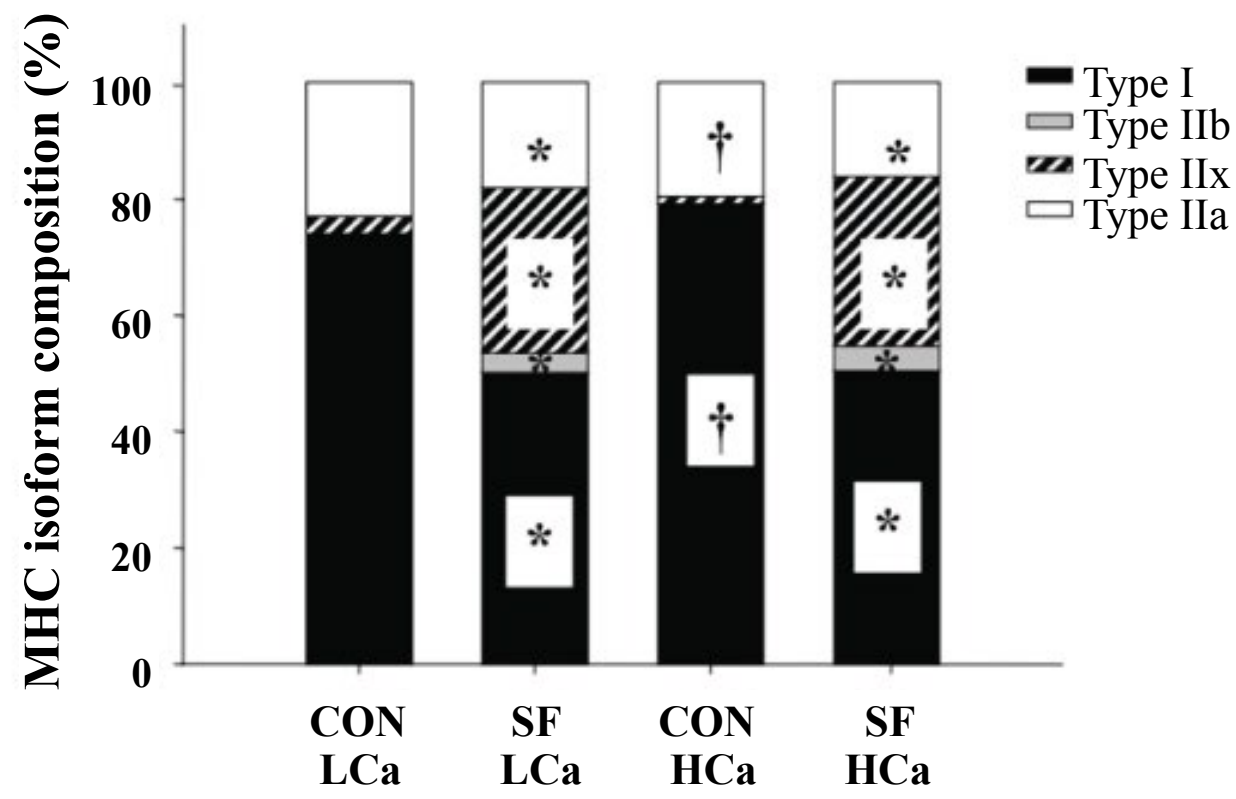

Figure 3: The composition of myosin heavy chain (MHC) isoforms in soleus muscle for flight (SF) and control (COn) animals on high-(HCa) and low-calcium (LCa) diets Significant difference between flight and control groups: ${ }^{*} \mathrm{P}<0.05$. Significant different between diet group: ${ }^{*} \mathrm{P}<0.05$. 
Citation: Taguchi S, Ogoh S, Hashimoto T, Yamasaki S, Okamoto H, et al. (2016) Spaceflight and Biohistochemical Alterations of Antigravity Soleus Muscle in Spontaneously Hypertensive Rats (SHR) to Dietary Low and High-Calcium Intake. J Bioanal Biomed 8: 041-047. doi:10.4172/1948593X.1000151

(A)

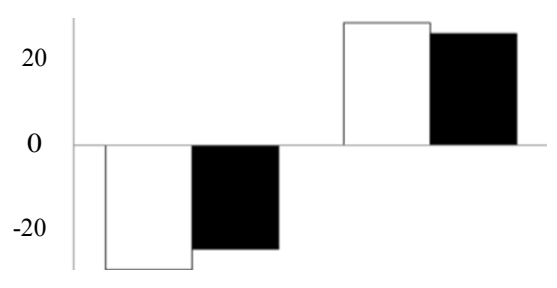

Type I Type IIx

The change of MHC isoform composition (\%)

(B)

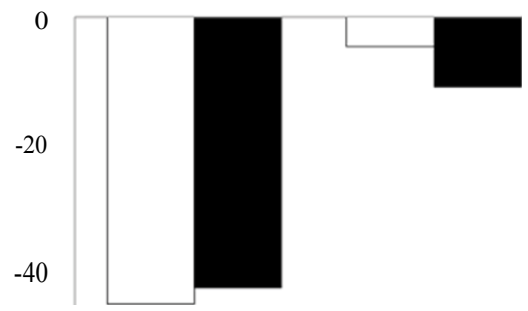

Type I Type IIa

$\%$ change of CSA $(\%)$

(C)

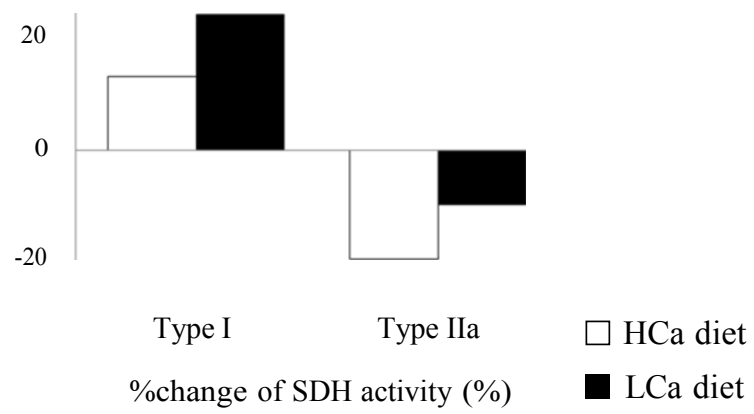

Figure 4: The change of MHC isoform composition (A), Cross sectional area (CSA) (B) and succinate dehydrogenase (SDH) activity of a single muscle fiber (C) on high-( $\mathrm{HCa})$ and lowcalcium (LCa) diets after spaceflight.

induced blood pressure differences in SHR [32]. Thus, it is possible that factors related to the calcium diet-induced blood pressure differences supported the difference in the proportion of type I MHC between the $\mathrm{HCa}$ and the LCa diet groups.

After spaceflight, a further increased fast-twitch fiber percent distribution counterbalanced by a decrease in slow-twitch fibers was observed in SHR soleus muscle. The degree of change in fiber type distribution in the soleus muscle from SHR after spaceflight was not different from the normotensive rats in a previous report [33], suggesting that the mechanism underlying this conversion in spaceflight may not involve hypertension-derived physiological factors. Shenkman and Nemirovskaya suggested that resting $\mathrm{Ca}^{2+}$ accumulation in myofibers may be one of the stimuli activating the slow-to-fast transition of MHC under unloading [13]. Although we did not measure intracellular $\mathrm{Ca}^{2+}$ accumulation in this study, the results suggest that the effect of dietary calcium on cellular $\mathrm{Ca}^{2+}$ metabolism and hence MHC transition might be smaller than those induced by spaceflight.

\section{Cross-sectional area (CSA) of a single muscle fiber}

In the present study, CSAs of single muscle fibers in soleus muscle were not significantly modified by different calcium diets in either the control or the spaceflight groups.

After spaceflight, there was a significant reduction of CSA in both diet groups. In humans following a 17-day flight, the fast type IIa fiber's CSA declined by $26 \%$ compared with a $15 \%$ reduction in CSA of the slow Type I fibers in soleus muscle [34]. Shenkman and Nemirovskaya suggested that soleus fibers overloading with $\mathrm{Ca}^{2+}$ under gravitational unloading leads to he significant cytoskeletal proteins degradation by 
Citation: Taguchi S, Ogoh S, Hashimoto T, Yamasaki S, Okamoto H, et al. (2016) Spaceflight and Biohistochemical Alterations of Antigravity Soleus Muscle in Spontaneously Hypertensive Rats (SHR) to Dietary Low and High-Calcium Intake. J Bioanal Biomed 8: 041-047. doi:10.4172/1948593X.1000151

showing that nifedipine (L-type $\mathrm{Ca}^{2+}$ channels blocker) administration inhibited the atrophy of Type I and Type II fibers under unloading $[12,13]$. However, the atrophy levels of a single muscle fiber were not influenced by different dietary calcium in either Type I or Type IIa fibers. These results suggested that dietary calcium might not have enough impact on the changes in cellular $\mathrm{Ca}^{2+}$ metabolism and thus could not inhibit the amount of atrophy of a single muscle fiber, which is strongly induced by spaceflight. This may be the case even though we have previously reported that dietary calcium altered bone mineral density as well as calcium metabolism in these animals [17]

\section{Histochemical enzyme activity of a single muscle fiber}

In both control and spaceflight SHR rats, the SDH activities of single muscle fibers were lower in the HCa diet groups than in the LCa diet groups. There was also a difference in alteration of SDH activity after spaceflight between the $\mathrm{LCa}$ and the $\mathrm{HCa}$ diet groups (Table 2 and Figure $4 \mathrm{C})$. It is likely that the increase of protease $\left(\mathrm{Ca}^{2+}\right.$-activated neural protease or cathepsin) was caused by the high calcium diet [35]. An elevation of the $\mathrm{Ca}^{2+}$ level in muscle fiber destroys mitochondria [36]. In addition, a cellular $\mathrm{Ca}^{2+}$ overload causes loss of mitochondrial potential and cell death [37]. Though the mechanism for the reduction of SDH activity is unclear, it is not to be denied that there was a dietary calcium change of the oxidative metabolism in soleus regardless of spaceflight. These results suggest the possibility that dietary calcium may inhibit the alteration of oxidative metabolism of muscle fiber during spaceflight.

Increases in the SDH activity of slow-twitch fibers from the soleus muscle were caused by spaceflight in both calcium diet groups. Conversely, the SDH activity of the FOG (Type IIa) fiber was reduced by spaceflight in both calcium diet groups. Fell et al., reported that 7 days of hindlimb suspension reduced the activity of citrate synthase to $83 \%$ of the control value in the soleus muscle [38]. Flynn and Max also found a qualitatively similar result after 13 days of hindlimb suspension [39]. Our observation that spaceflight increased the SDH activity of Type I fibers appears to conflict with these finding on whole muscles. However, Fitts et al., reported results similar to our findings and showed that hindlimb suspension increased the specific activity of citrate synthase in Type I fibers from the soleus muscle [40]. They suggested that the nonmitochondrial cell protein decreased to a greater extent than the mitochondrial fraction. Booth reported that cytochrome $\mathrm{c}$ and citrate synthase in the gastrocnemius decayed faster than the loss of muscle weight in the first 4 days of hindlimb immobilization [41]. Consequently, the mitochondrial potential reached their new steady-state levels while muscle weight was still decreasing. Riley et al., reported that subsarcolemmal mitochondria were preferentially lost and reduced below normal concentration [42]. However, the enzyme activity after spaceflight depended on the level of muscle atrophy and the reduction of mitochondrial potential. It is possible that the absolute mitochondrial content decreased, but the relatively greater breakdown of myofibrillar proteins elevated mitochondrial concentration in the muscle fiber. Thus, the large atrophy of CSA could explain our finding of an increased SDH activity in Type I fiber after spaceflight. On the other hand, it is likely that the influence of the absolute mitochondrial content decreased upon reduced SDH activity in Type IIa fiber after spaceflight because of the small CSA reduction.

Bonen et al., reported that microgravity increased insulinstimulated glucose transport [43]. This enhanced insulin- glucose transport activity is likely caused by the development of increased insulin binding [43] as well as by a roughly proportional increase in the expression of GLUT4 protein [44]. The GPD activities of both SO and
FOG fibers were increased after spaceflight in both the HCa and the LCa diet groups. These results suggest that the absolute mitochondrial content significantly decreased [42] and was accompanied by an increase in glycolytic metabolism in the muscle fiber after spaceflight. However, the change in glycolytic metabolism of the muscle fiber after spaceflight was not influenced by dietary calcium. Thus, it is not likely that the change in glycolytic metabolism was parallel to the alteration in oxidative metabolism.

In conclusion, spaceflight caused an increased fast-twitch fiber percent distribution counterbalanced by a decrease in slow-twitch fibers. Slow-twitch fibers atrophied more than fast-twitch fiber in SHR soleus muscle. However, dietary calcium levels did not influence these alterations of fiber type distribution and cross-sectional area that were caused by spaceflight. On the other hand, the oxidative enzyme (SDH) activity was influenced by both spaceflight and dietary calcium. Increases in the SDH activity of slow-twitch fiber from soleus muscle were caused by spaceflight. Conversely, the SDH activity of Type IIa fiber was reduced by spaceflight. In addition, the mean SDH activities of a single muscle fiber were lower in the HCa diet than the LCa diet groups in both the control and the spaceflight rats. These results suggest the possibility that the effect of dietary calcium on fiber type composition and fiber size may be smaller than that of spaceflight, while dietary calcium could inhibit the alteration of oxidative metabolism of muscle fiber on spaceflight in SHR.

Sometimes astronauts have to perform a long-duration space mission, while reduction of soleus muscle mass was less evident compared with rodents, possibly via exercise countermeasures [45]. Further studies are needed to elucidate beneficial effects of resistance exercise and nutritional support (e.g., dietary calcium) against longduration microgravity.

\section{Acknowledgement}

This work was supported in part by National Aeronautics and Space Administration Grant no. NCC-2-934 and the Grant-in-Aid from the Japan Society from Promotion of Science (\#25560380, \#25282201). We also thank Dr. Nobumasa Iwanaka for help with the data analysis.

\section{References}

1. Bassett DR (1994) Skeletal muscle characteristics: relationships to cardiovascular risk factors. Med Sci Sports Exerc 26: 957-966.

2. Ben BLL, Sempore B, Mayet MH, Favier RJ (1990) Evidence of a slow-to-fast fiber type transition in skeletal muscle from spontaneously hypertensive rats. Am J Physiol 258: 352-357.

3. Benbachir LL, Koubi H, Sempore B, Mayet MH, Frutoso J, et al. (1993) Soleus muscle alterations in spontaneously hypertensive rats are not dependent on activation of beta 2-adrenergic receptors. J Auton Nerv Syst 44: 161-170.

4. Lewis DM, Levi AJ, Brooksby P, Jones JV (1994) A faster twitch contraction of soleus in the spontaneously hypertensive rat is partly due to changed fibre type composition. Exp Physiol 79: 377-386.

5. Massie BM, Simonini A, Sahgal P, Wells L, Dudley GA (1996) Relation of systemic and local muscle exercise capacity to skeletal muscle characteristics in men with congestive heart failure. J Am Coll Cardiol 27: 140-145.

6. Doyle F, Brown J, Lachance C (1970) Relation between bone mass and muscle weight. Lancet 1: 391-393.

7. Baptista F, Barrigas C, Vieira F, Santa Clara H, Homens PM, et al. (2012) The role of lean body mass and physical activity in bone health in children. $J$ Bone Miner Metab 30: 100-108.

8. Miller LE, Pierson LM, Pierson ME, Kiebzak GM, Ramp WK et al. (2009) Age influences anthropometric and fitness-related predictors of bone mineral in men. Aging Male 12: 47-53

9. Ogoh S, Yamasaki S, Okamoto H, Fukuda S, Yamori S, et al. (1995) Effect of weightlessness simulation on skeletal muscle histochemistry and bone 
Citation: Taguchi S, Ogoh S, Hashimoto T, Yamasaki S, Okamoto H, et al. (2016) Spaceflight and Biohistochemical Alterations of Antigravity Soleus Muscle in Spontaneously Hypertensive Rats (SHR) to Dietary Low and High-Calcium Intake. J Bioanal Biomed 8: 041-047. doi:10.4172/1948593X.1000151

metabolism of spontaneously hypertensive rats. Nihon Seirigaku Zasshi 57: 419-426.

10. Ingalls CP, Warren GL, Armstrong RB (1999) Intracellular $\mathrm{Ca}^{2+}$ transients in mouse soleus muscle after hindlimb unloading and reloading. J Appl Physio 87: 386-390.

11. Pardo AC, Rinaldi GJ, Mosca SM (2015) Mitochondrial calcium handling in normotensive and spontaneously hypertensive rats: correlation with systolic blood pressure levels. Mitochondrion 20: 75-81.

12. Mukhina AM, Altaeva EG, Nemirovskaya TL, Shenkman BS (2008) The role of L-type calcium channels in the accumulation of $\mathrm{Ca} 2+$ in soleus muscle fibers in the rat and changes in the ratio of myosin and serca isoforms in conditions of gravitational unloading. Neurosci Behav Physiol 38: 181-188.

13. Shenkman BS, Nemirovskaya TL (2008) Calcium-dependent signaling mechanisms and soleus fiber remodeling under gravitational unloading. J Muscle Res Cell Motil 29: 221-230.

14. Hansford RG (1994) Role of calcium in respiratory control. Med Sci Sports Exerc 26: 44-51.

15. Hamet $P$ (1995) The evaluation of the scientific evidence for a relationship between calcium and hypertension. J Nutr 125: 311S-400S.

16. McCarron DA (1998) Diet and blood pressure the paradigm shift. Science 281 933-934.

17. Hatton DC, Yue Q, Dierickx J, Roullet C, Otsuka K, et al. (2002) Calcium metabolism and cardiovascular function after spaceflight. J Appl Physiol 92: 3-12.

18. McCarron DA, Morris CD, Henry HJ, Stanton JL (1984) Blood pressure and nutrient intake in the United States. Science 224: 1392-1398.

19. Ono A, Kuwaki T, Cao WH, Kumada M, Fujita T (1994) High calcium die prevents baroreflex impairment in salt-loaded spontaneously hypertensive rats. Hypertension 24: 83-90.

20. Mabuchi K, Sreter FA (1980) Actomyosin ATPase II Fiber typing by histochemical ATPase reaction. Muscle Nerve 3: 233-239.

21. Peter JB, Barnard RJ, Edgerton VR, Gillespie CA, Stempel KE (1972) Metabolic profiles of three fiber types of skeletal muscle in guinea pigs and rabbits. Biochemistry 11: 2627-2633.

22. Nachlas MM, Miller DI, Siedband MP (1966) Continuous monitoring of patients with acute myocardial infarction. A 32-month experience. JAMA 198: 119-126.

23. Desplanches D, Mayet MH, Sempore B, Flandrois R (1987) Structural and functional responses to prolonged hindlimb suspension in rat muscle. J Appl Physiol 63: 558-563.

24. Talmadge RJ, Roy RR (1993) Electrophoretic separation of rat skeletal muscle myosin heavy-chain isoforms. J Appl Physiol 75: 2337-2340.

25. Hashimoto T, Kambara N, Nohara R, Yazawa M, Taguchi S (2004) Expression of MHC-beta and MCT1 in cardiac muscle after exercise training in myocardialinfarcted rats. J Appl Physiol 97: 843-851.

26. Diffee GM, Caiozzo VJ, Herrick RE, Baldwin KM (1991) Contractile and biochemical properties of rat soleus and plantaris after hindlimb suspension. Am J Physiol 260: 528-534

27. Fitts RH, Romatowski JG, Blaser C, De La Cruz L, Gettelman GJ, et al. (2000) Effect of spaceflight on the isotonic contractile properties of single skeletal muscle fibers in the rhesus monkey. J Gravit Physiol 7: S53-S54.

28. Ohira Y, Yoshinaga T, Nonaka I, Ohara M, Yoshioka T, et al. (2000) Histochemica responses of human soleus muscle fibers to long-term bedrest with or without countermeasures. Jpn J Physiol 50: 41-47.

29. Schuenke MD, Reed DW, Kraemer WJ, Staron RS, Volek JS, et al. (2009) Effects of 14 days of microgravity on fast hindlimb and diaphragm muscles of the rat. Eur J Appl Physiol 106: 885-892.

30. Bortolotto SK, Stephenson DG, Stephenson GM (1999) Fiber type populations and $\mathrm{Ca}^{2+}$-activation properties of single fibers in soleus muscles from $\mathrm{SHR}$ and WKY rats. Am J Physiol 276: C628-C637.

31. Mondon CE, Reaven GM, Azhar S, Lee CM, Rabkin R (1989) Abnormal insulin metabolism by specific organs from rats with spontaneous hypertension. Am J Physiol 257: E491-E498.

32. Hatton DC, McCarron DA (1994) Dietary calcium and blood pressure in experimental models of hypertension. A review Hypertension 23: 513-530.

33. Desplanches D, Mayet MH, llyina Kakueva El, Sempore B, Flandrois R (1990) Skeletal muscle adaptation in rats flown on Cosmos 1667. J Appl Physiol 68 : 48-52.

34. Widrick JJ, Knuth ST, Norenberg KM, Romatowski JG, Bain JL, et al. (1999) Effect of a 17 day spaceflight on contractile properties of human soleus muscle fibres. J Physiol 516: 915-930.

35. Dougherty RM, lacono JM (1979) Effects of dietary calcium on blood and tissue lipids, tissue phospholipids, calcium and magnesium levels in rabbits fed diets containing beef tallow. J Nutr 109: 1934-1945.

36. Kristian T, Siesjo BK (1998) Calcium in ischemic cell death. Stroke 29: 705-718.

37. Duchen MR (2000) Mitochondria and $\mathrm{Ca}^{2+}$ in cell physiology and pathophysiology. Cell Calcium 28: 339-348.

38. Fell RD, Steffen JM, Musacchia XJ (1985) Effect of hypokinesia-hypodynamia on rat muscle oxidative capacity and glucose uptake. Am J Physiol 249: R308-R312.

39. Flynn DE, Max SR (1985) Effects of suspension hypokinesia/hypodynamia on rat skeletal muscle. Aviat Space Environ Med 56: 1065-1069.

40. Fitts RH, Brimmer CJ, Heywood Cooksey A, Timmerman RJ (1989) Single muscle fiber enzyme shifts with hindlimb suspension and immobilization. Am J Physiol 256: C1082-C1091.

41. Booth FW (1977) Time course of muscular atrophy during immobilization of hindlimbs in rats. J Appl Physiol Respir Environ Exerc Physiol 43: 656-661.

42. Riley DA, Ellis S, Giometti CS, Hoh JF, llyina Kakueva El, et al. (1992) Muscle sarcomere lesions and thrombosis after spaceflight and suspension unloading J Appl Physiol 73: S33-S43.

43. Bonen A, Elder GC, Tan MH (1988) Hindlimb suspension increases insulin binding and glucose metabolism. J Appl Physiol 65: 1833-1839.

44. Henriksen EJ, Rodnick KJ, Mondon CE, James DE, Holloszy JO (1991) Effect of denervation or unweighting on GLUT-4 protein in rat soleus muscle. J App Physiol 70: 2322-2327

45. Sandona D, Desaphy JF, Camerino GM, Bianchini E, Ciciliot S, et al (2012) Adaptation of mouse skeletal muscle to long-term microgravity in the MDS mission. PLoS One 7: e33232. 